\title{
Supporting Information \\ Modulation of a mycobacterial ADP-ribosyltransferase to augment rifamycin antibiotic resistance
}

\section{Authors:}

Meng Zheng, Tania J. Lupoli ${ }^{*}$

Affiliation:

Department of Chemistry, New York University, New York, NY 10003, United States

${ }^{*}$ Corresponding author 
Table S1. Primers used in this study.

\begin{tabular}{cll} 
No. & Name & Sequence \\
\hline 1 & Msm_Arr_fow & GAGGCTCACAGAGAACAGATTGGTGGGGTGGCGAATCCGCC \\
& GAAAC & CTTTCGGGCTTTGTTAGCAGCCGGATCAGCTAGTCATAGATGA \\
2 & Msm_Arr_rev & CCGCCAGCCC \\
3 & Msm_Arr_N38A_fow & CGGCCGCGAGTCCAACTTCGAGGCCGGGCGCATCATGAACC \\
4 & Msm_Arr_N38A_rev & GGTTCATGATGCGCCCGGCCTCGAAGTTGGACTCGCGGCCG \\
5 & Msm_Arr_F39A_fow & CGCGAGTCCAACTTCGAGGCCGGGCGCATCATGAACC \\
6 & Msm_Arr_F39A_rev & GGTTCATGATGCGCCCGGCCTCGAAGTTGGACTCGCG
\end{tabular}

Table S2. Plasmids used in this study.

\begin{tabular}{|c|c|c|c|}
\hline No. & Plasmid name & Description & Source \\
\hline 1 & pETHisSUMO & pET His 6 SUMO TEV LIC cloning vector $\left(a^{2} p^{R}\right)$ & Addgene \#29711* \\
\hline 2 & pHYRS52 & His6-S. cerevisiae Ulp1 (res. 403-621) $\left(\mathrm{amp}^{\mathrm{R}}\right)$ & Addgene $\# 31122^{\star * 1}$ \\
\hline 3 & TL143 & $\begin{array}{l}\text { pET His SUMO Msm Arr (Msm 1221); constructed with } \\
\text { plasmid } 1 \text { and primers } 1 \text { and } 2\end{array}$ & This work \\
\hline 4 & MZ295 & $\begin{array}{l}\text { pET His SUMO Msm Arr N38A; constructed with plasmid } 3 \text { and } \\
\text { primers } 3 \text { and } 4\end{array}$ & This work \\
\hline 5 & MZ296 & $\begin{array}{l}\text { pET His SUMO Msm Arr F39A; constructed with plasmid } 3 \text { and } \\
\text { primers } 5 \text { and } 6\end{array}$ & This work \\
\hline \multicolumn{4}{|c|}{$\begin{array}{l}\text { *pET His6 Sumo TEV LIC cloning vector (2S-T) was a gift from Scott Gradia (Addgene plasmid } \\
\text { \# 29711; http://n2t.net/addgene:29711 ; RRID:Addgene_29711) }\end{array}$} \\
\hline
\end{tabular}


Table S3. Strains used in this study.

\begin{tabular}{|c|c|c|c|}
\hline No. & Strain name & Description & Source \\
\hline 1 & Rosetta2 & E. coli BL21 derivative for rare codon usage & Novagen \\
\hline 2 & Msm mc 2155 & Lab wild type strain of $M$. smegmatis & $\begin{array}{l}\text { Gift from } \\
\text { Glickman Lab }\end{array}$ \\
\hline 3 & EcTL143 & E. coli Rosetta2 expressing TL143 & This work \\
\hline 4 & EcMZ295 & E. coli Rosetta2 expressing MZ295 & This work \\
\hline 5 & EcMZ296 & E. coli Rosetta2 expressing MZ296 & $\begin{array}{l}\text { This work } \\
\text { Stalling et. al. }\end{array}$ \\
\hline 6 & MGM1706 & M. smegmatis $\Delta$ arr & $\begin{array}{l}2011^{2} \\
\text { Stalling et. al. }\end{array}$ \\
\hline 7 & MGM3051 & M. smegmatis $\Delta$ arr $:: P m o p$-flag-arr $\left(\right.$ hyg $\left.^{\mathrm{R}}\right)$ & 2011 \\
\hline 8 & M. abscessus & Mycobacterium abscessus (NR-44263) & $\mathrm{BEl}$ \\
\hline 9 & BW25113 $\Delta \Delta$ & E. coli BW25113 $\Delta$ bamB $\Delta t o / C$ & Cox et. al. $2017^{3}$ \\
\hline 10 & BW25113 $\Delta \Delta$ Arr & E. coli BW25113 $\triangle$ bamBAtolC ::pGDP4-arr & Cox et. al. 2017 \\
\hline
\end{tabular}




\section{Supplementary Figures}
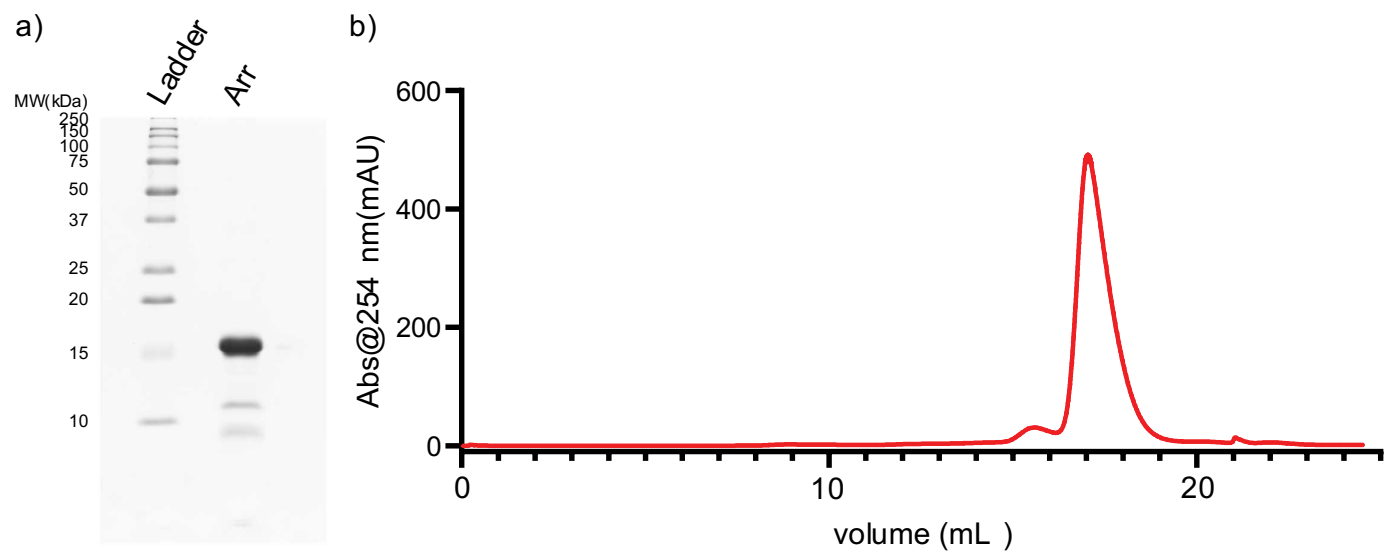

Figure S1. Characterization of recombinant Msm Arr protein a) SDS-PAGE of purified Msm Arr (18\% acrylamide gel); b) Gel-filtration chromatography trace of purified Msm Arr.
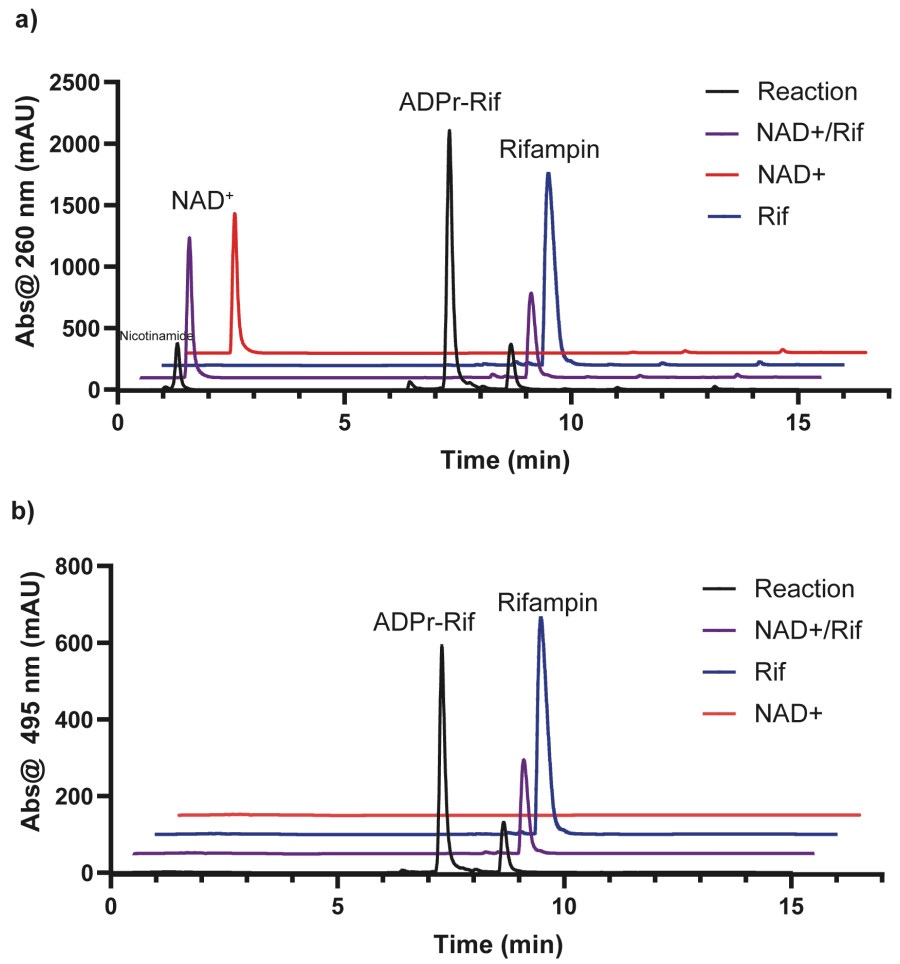

Figure S2. HPLC trace of rifampin ADP-ribosylation reaction catalyzed by Arr. Indicated reactions monitored by absorbance a) at $260 \mathrm{~nm}$ and b) $495 \mathrm{~nm}$. Red trace: only NAD+ in reaction buffer; Blue trace: only rifampin in reaction buffer; Purple trace: both NAD+ and rifampin in reaction buffer; Black trace (Reaction): NAD and rifampin with Arr in reaction buffer after 30 minutes incubation. Concentrations are indicated in the Methods section. 


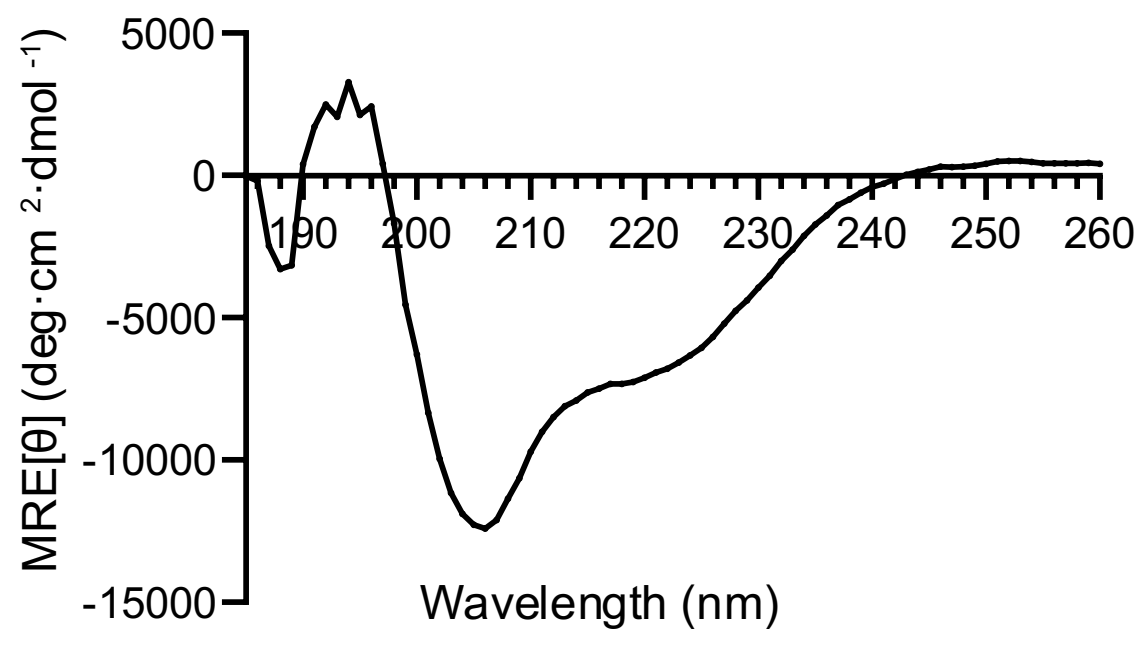

Figure S3. Circular dichroism spectrum of Arr ${ }^{\mathrm{C}}$. Peptide $(50 \mu \mathrm{M})$ was incubated in 50 $\mathrm{mM}$ Tris- $\mathrm{HCl}(\mathrm{pH} 7.5)$ buffer at $25^{\circ} \mathrm{C}$. Measurement was made on a Jasco J-1500 CD Spectrometer $(0.1 \mathrm{~cm}$ sample cell length).

a)

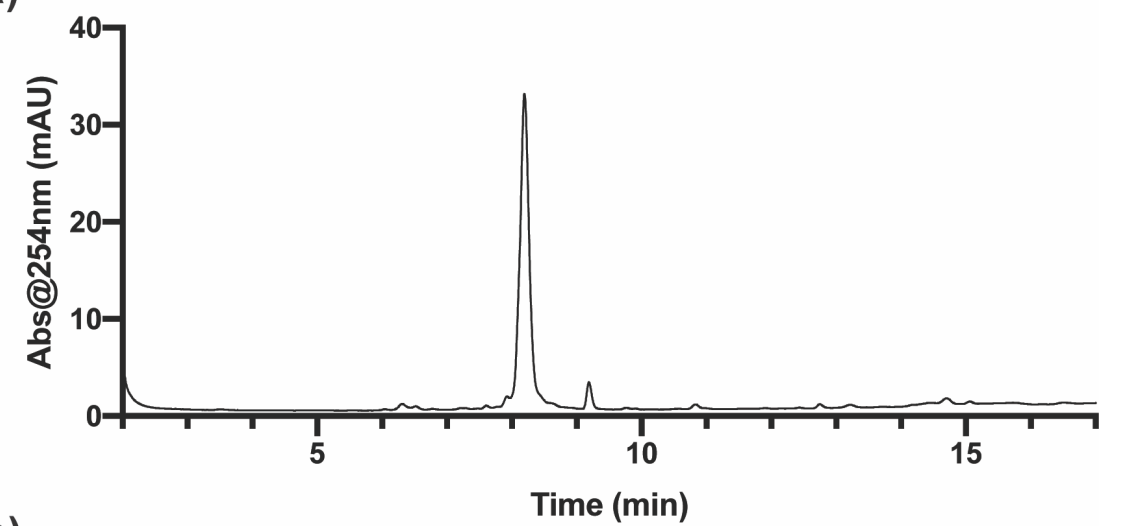

b)

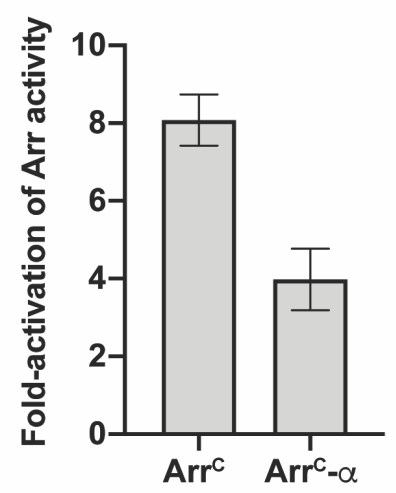

Figure S4. Characterization of a truncation of Arr' containing the alpha-helical region demonstrates partial activation of Arr. a) Analytical HPLC analysis of ArrC alpha (sequences shown in Figure 2A). b) Fold activation of Arr (250 nM) with $5 \mu \mathrm{M} \mathrm{ArrC}$ or Arr ${ }^{\mathrm{C}}$-alpha ( $\mathrm{t}=5 \mathrm{~min}$ at $37^{\circ} \mathrm{C}, 100 \mu \mathrm{M}$ NAD+, $100 \mu \mathrm{M}$ rifampin, 10 min pre-incubation). $(n=2-3$ with standard deviation (SD) indicated by bars.) 
a)

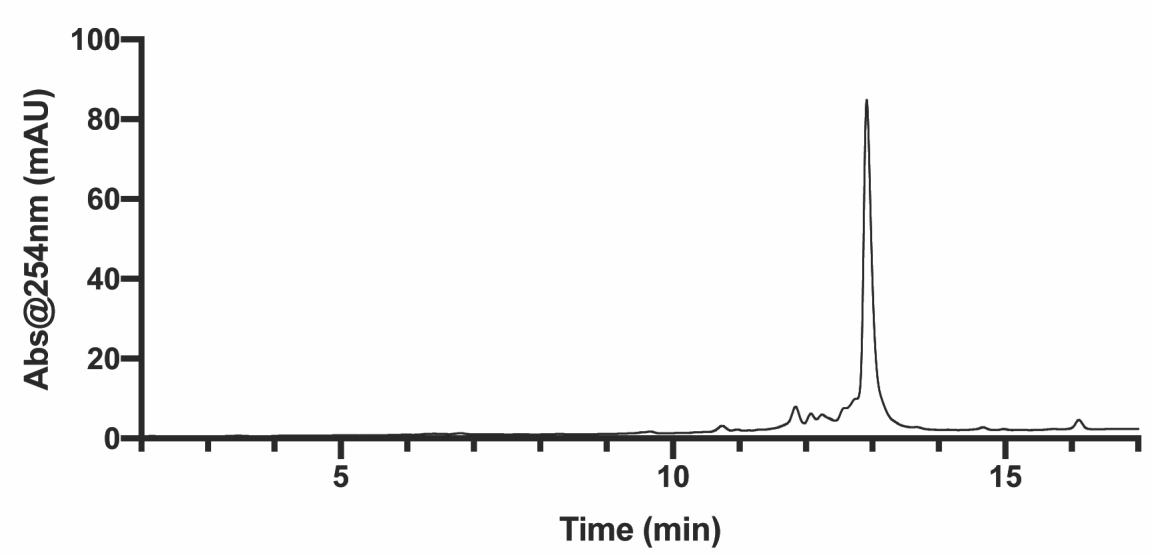

b)

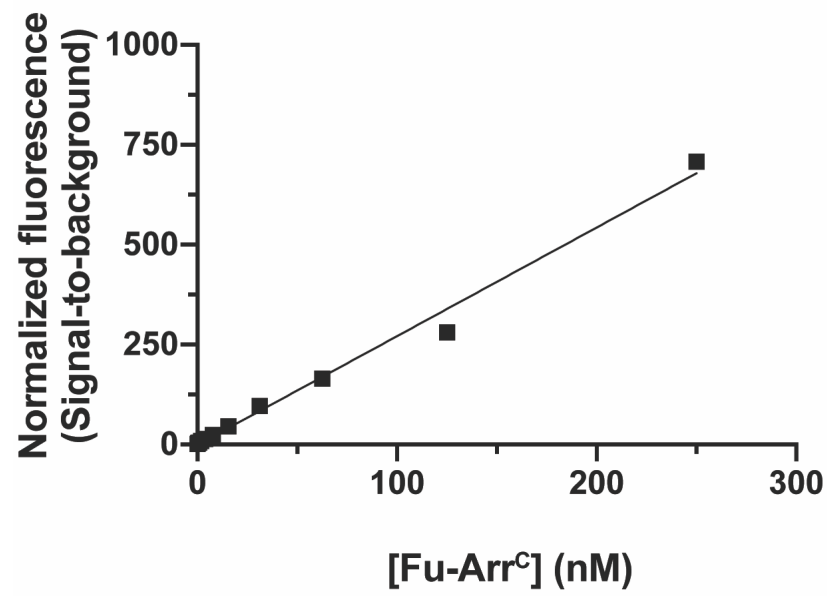

c)

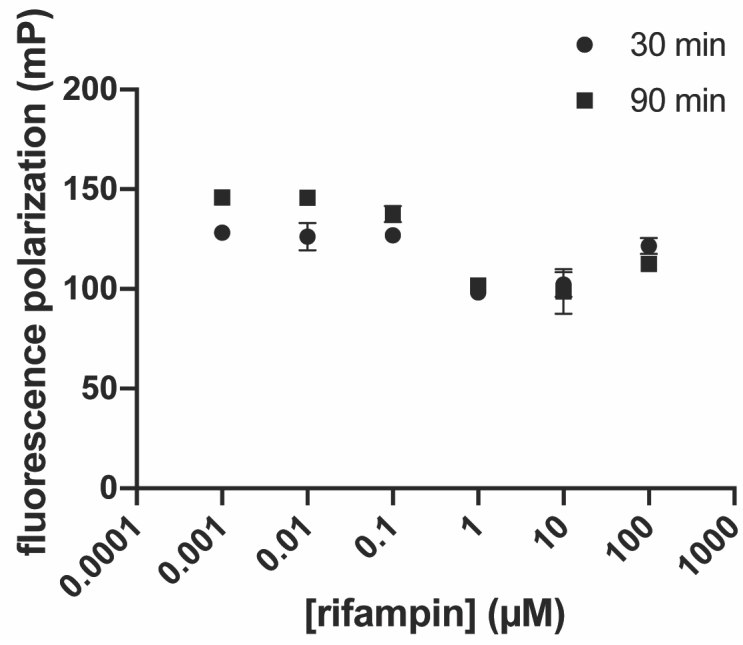

Figure S5. Characterization of a fluorescent $\mathrm{Arr}^{\mathrm{C}}$ probe for binding experiments indicates no direct interaction with rifampin. a) Analytical HPLC analysis of fluorescein-labeled $\operatorname{Arr}^{C}(F u-A r r C)$. b) Signal-to-background measurement of increasing concentration of Fu-ArrC; concentration of probe was chosen with ratio $>50$. c) Direct binding experiment measuring fluorescence polarization with increasing concentration of rifampin and $\mathrm{Fu}-\mathrm{Arr}^{\mathrm{C}}(25 \mathrm{nM})$ suggests no interaction at indicated incubation times. $\mathrm{mP}=$ milipolarization. ( $\mathrm{n}=3$ for part $\mathrm{C}$ with $\mathrm{SD}$ indicated by bars.) 


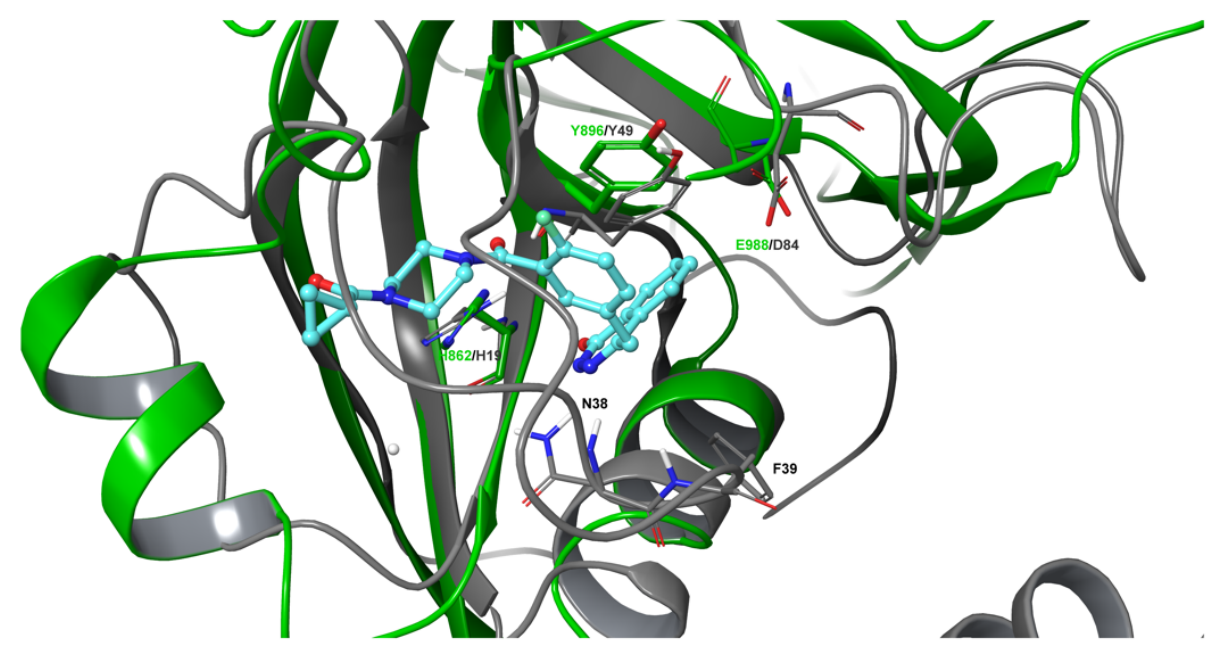

Figure S6. Structural alignment of Msm Arr (PDB ID: 2HW2) and hPARP-1 catalytic domain (PDB ID: 7AAD, with Olaparib bound). The side chain of key residues are highlighted in grey (Msm Arr: His19-Tyr49-Asp84) and in green (PARP1: His862-Tyr896Glu988). Based on local alignment of the indicated catalytic residues in Glide, the calculated RMSD is 2.86 .

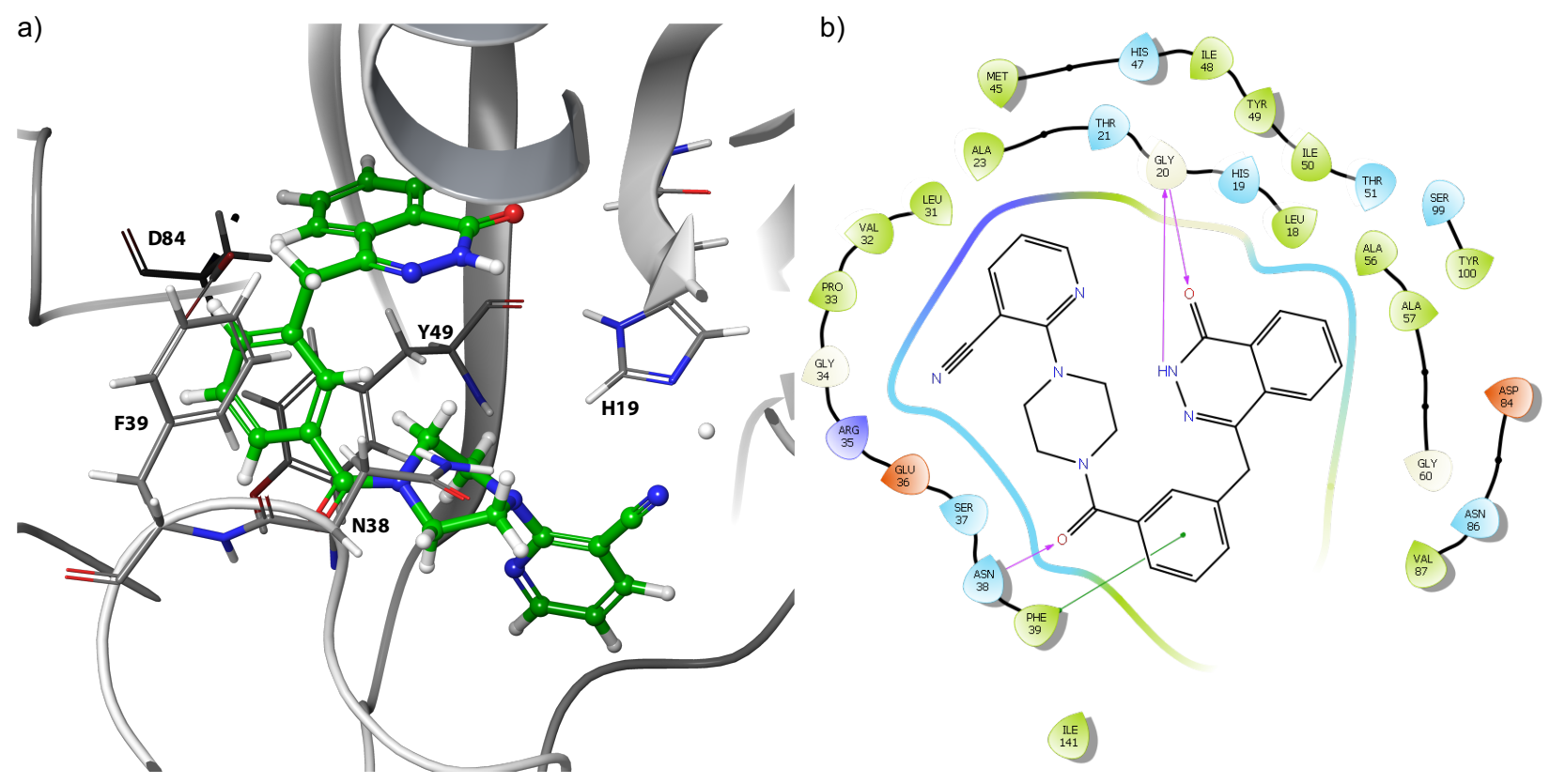

Figure S7. Docking of AZ9482 (10) into the NAD+ binding pocket of Msm Arr (PDB ID: 2HW2). a) Cartoon and stick representation of 10 bound to Msm Arr. Docking was performed as described in the Methods section and indicated pose was selected, which had the lowest Glide docking score (-9.677). b) Interaction map of pose from part A. Purple arrows indicate $\mathrm{H}$-bonds; green arrow indicates pi-pi stacking. 


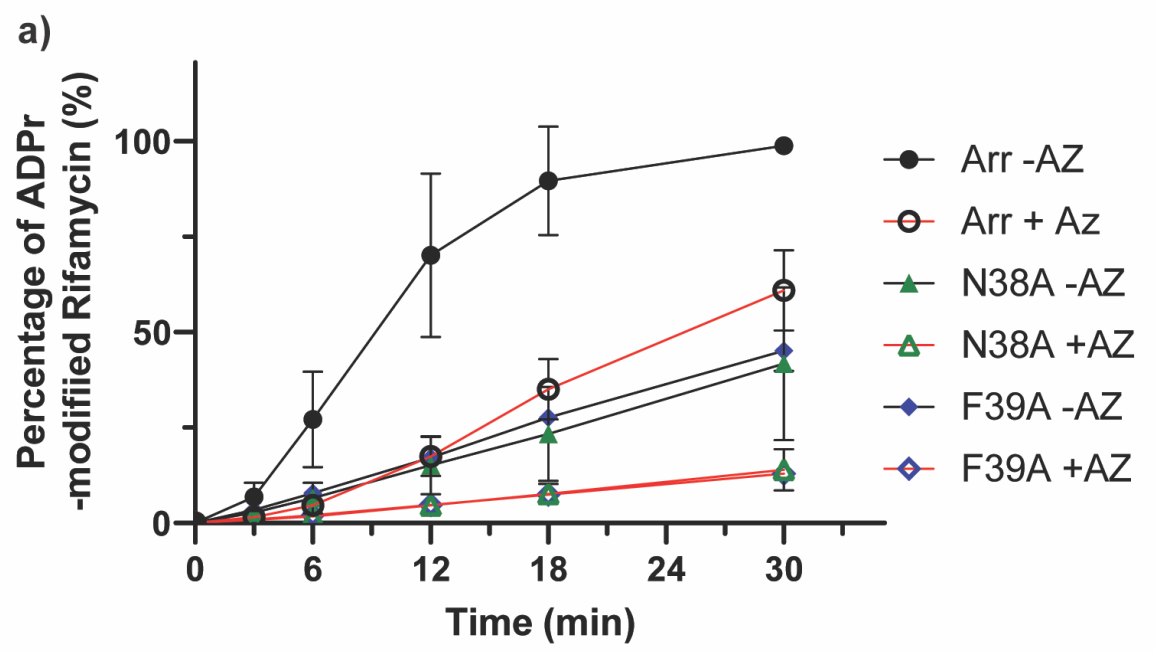

b)

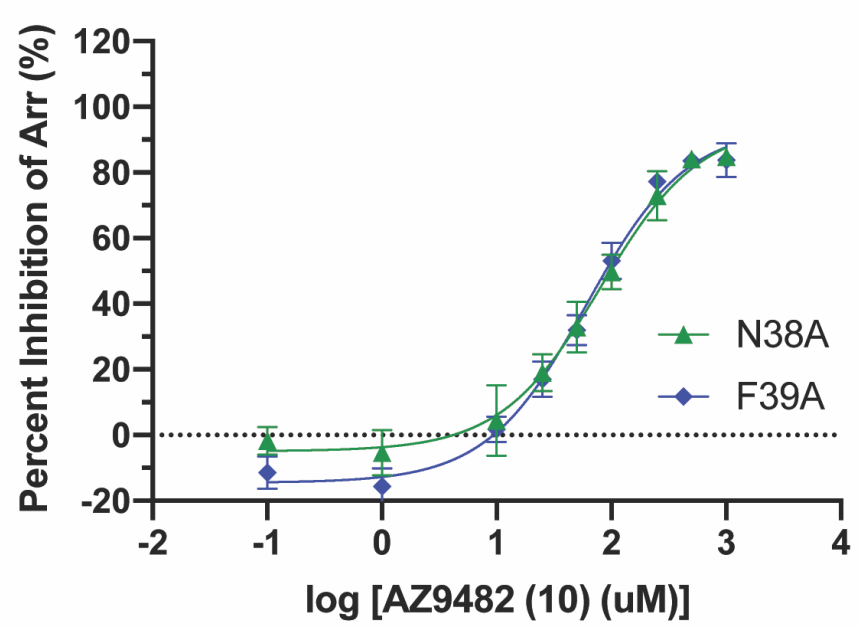

\begin{tabular}{c|c} 
mutant & IC $_{50}(\mu \mathbf{M})$ \\
\hline N38A & $80.5+/-24.3$ \\
F39A & $60.3+/-12.6$
\end{tabular}

Figure S8. Inhibition of Arr mutants by compound 10 (AZ9482) a) Time course of rifampin ADP-ribosylation catalyzed by Arr $(0.5 \mu \mathrm{M}$, black circles), Arr mutants $(5 \mu \mathrm{M}$, N38A green/F39A blue) with (red lines) or without (black lines) the addition of compound 10 (AZ); b) Dose-dependent inhibitory effect of compound 10 against Arr mutants (N38A/F39A) with $5 \mu \mathrm{M}$ enzyme ( $t=30$ min for each reaction). The 95 percent confidence intervals of the $\mathrm{IC}_{50}$ values are indicated. (For all experiments, $\mathrm{n}=3$, SD is indicated by bars.) 
a)
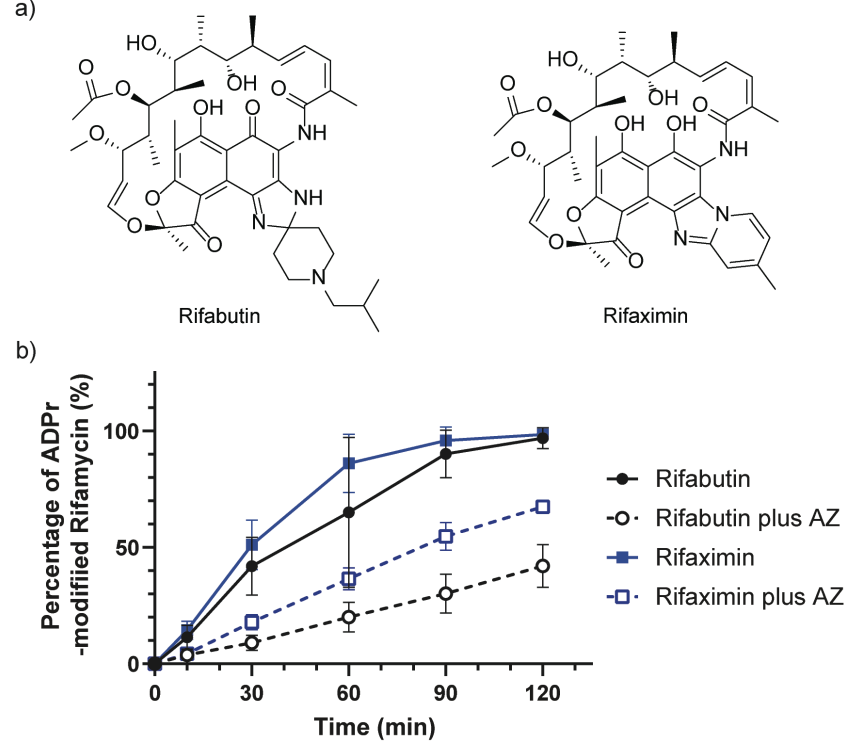

Figure S9. Inhibitory effect of AZ9482 on the ADP-ribosylation of rifabutin and rifaximin. a) Structures of rifabutin and rifaximin; b) Time course of ADP-ribosylation of rifabutin (black) and rifaximin (blue), with the addition of compound 10 (dashed lines) and without (solid lines). See Methods for detailed protocol, here [AZ9482] $=100 \mu \mathrm{M},[$ Arr $]=$ $0.5 \mu M$. ( $n=3, S D$ is indicated by bars.) 

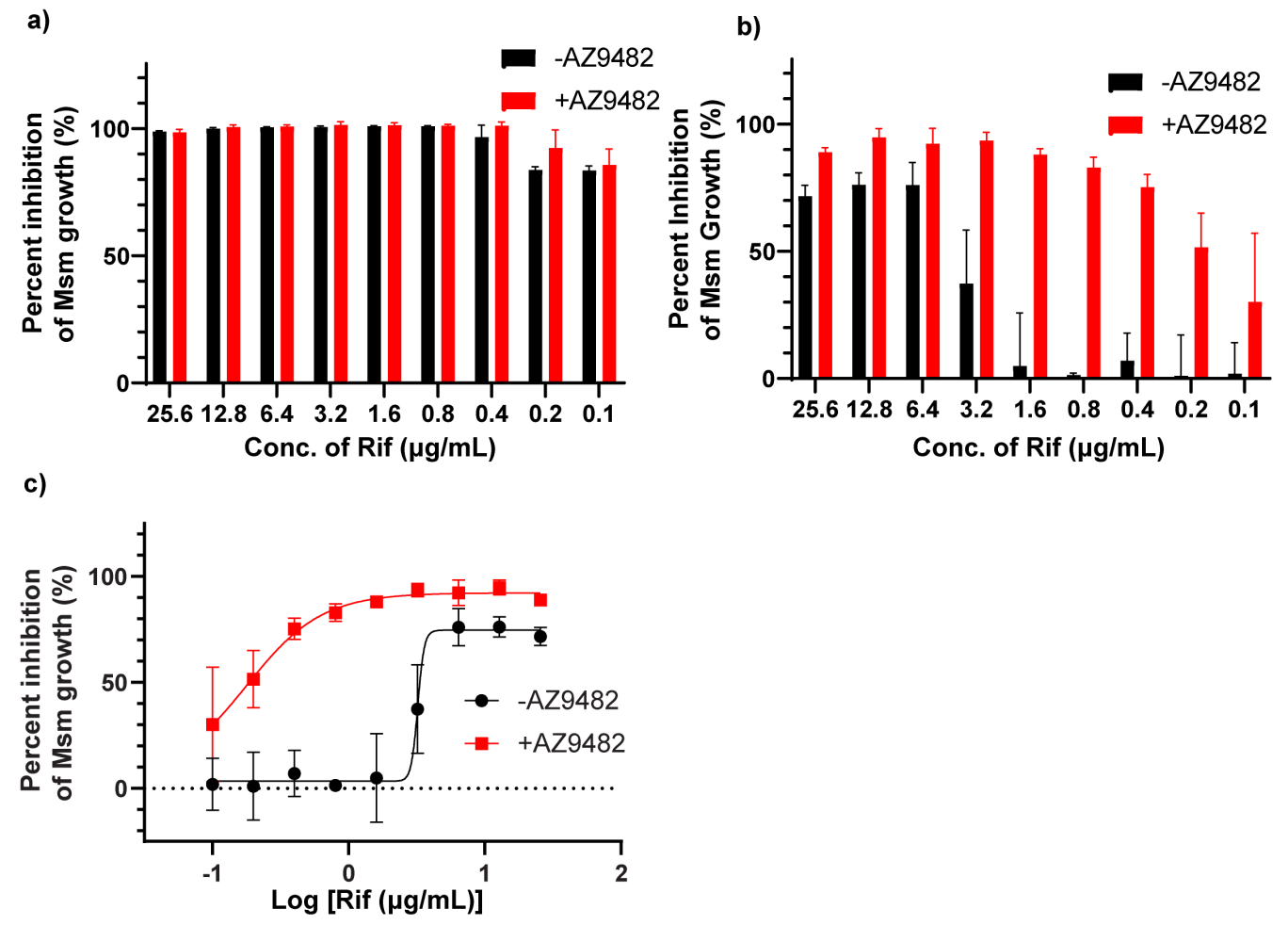

Figure S10. Arr is the cellular target of AZ9482 (10) in Msm. Growth inhibition of the following strains in the presence of increasing concentrations of rifampin (Rif) with (red) or without (black) $96 \mu \mathrm{g} / \mathrm{mL}$ AZ9482: a) Msm $\Delta$ arr (MGM1706) and b) Msm $\Delta a r r$ complementation strain (MGM3051). Data shown was measured on day 3. C) Minimum inhibitory concentration curves for Msm $\Delta$ arr complementation strain, based on data from part $B(n=3$, bars indicate $S D)$.

a)

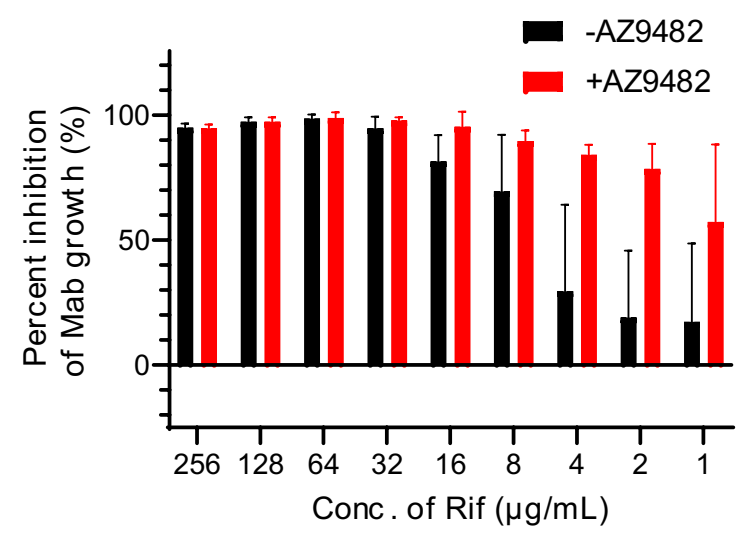

Figure S11. AZ9482 (10) is an effective Rifampin adjuvant in M. abscessus (Mab). Dose-dependent growth inhibitory effect of rifampin with (red) or without (black) $96 \mu \mathrm{g} / \mathrm{mL}$ AZ9482 against $M$. abscessus. (Data measured on day $5, \mathrm{n}=9, \mathrm{SD}$ indicated by bars.) 


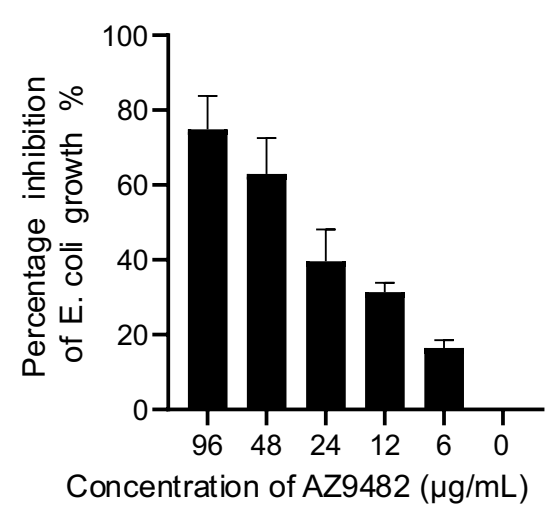

Figure S12. AZ9482 (10) is toxic against E. coli BW25113 $\Delta \Delta$. E. coli $\left(\mathrm{OD}_{600}=0.01\right)$ was incubated with corresponding concentrations of 10. (Data measured on day $2, n=3$, bars represent SD.)

a)

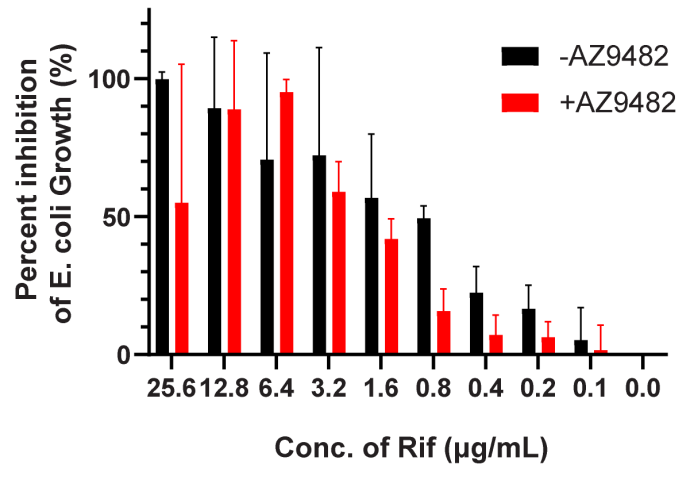

b)

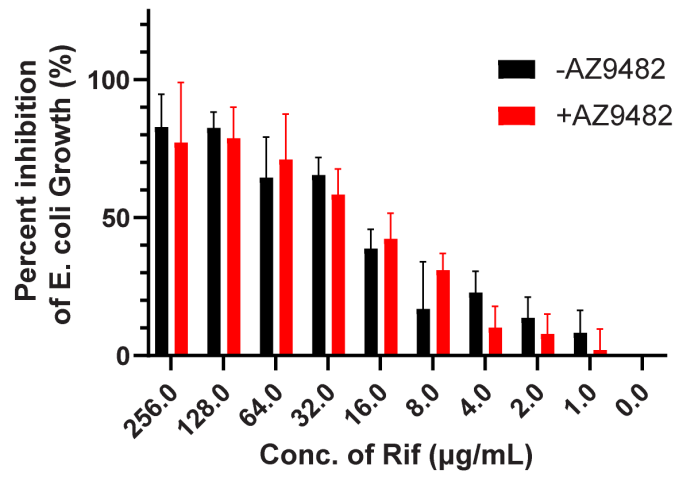

Figure S13. Nontoxic concentration of AZ9482 (10) does not potentiate Rifampin in E. coli. Dose dependent inhibitory effect of rifampin with (red) or without (black) $12 \mu \mathrm{g} / \mathrm{mL}$ AZ9482 against the following strains: a) E. coli BW25113 $\Delta \Delta$ and b) $E$. coli BW25113 $\Delta \Delta$ /pGDP4-arr. (Data measured on day $2, \mathrm{n}=6$, bars represent SD.) 


\section{References:}

1. Muona, M.; Aranko, A. S.; Iwai, H., Segmental isotopic labelling of a multidomain protein by protein ligation by protein trans-splicing. Chembiochem 2008, 9 (18), 2958-61.

2. Stallings, C. L.; Chu, L.; Li, L. X.; Glickman, M. S., Catalytic and non-catalytic roles for the mono-ADP-ribosyltransferase Arr in the mycobacterial DNA damage response. PLoS One 2011, 6 (7), e21807.

3. Cox, G.; Sieron, A.; King, A. M.; De Pascale, G.; Pawlowski, A. C.; Koteva, K.; Wright, G. D., A Common Platform for Antibiotic Dereplication and Adjuvant Discovery. Cell Chem Biol 2017, 24 (1), 98-109. 